\title{
Multicomponent Burgers and KP Hierarchies, and Solutions from a Matrix Linear System ${ }^{\star}$
}

\author{
Aristophanes DIMAKIS ${ }^{\dagger}$ and Folkert MÜLLER-HOISSEN ${ }^{\ddagger}$ \\ $\dagger$ Department of Financial and Management Engineering, \\ University of the Aegean, 31 Fostini Str., GR-82100 Chios, Greece \\ E-mail: dimakis@aegean.gr \\ $\ddagger$ Max-Planck-Institute for Dynamics and Self-Organization, \\ Bunsenstrasse 10, D-37073 Göttingen, Germany \\ E-mail: folkert.mueller-hoissen@ds.mpg.de
}

Received November 01, 2008, in final form January 04, 2009; Published online January 08, 2009 doi:10.3842/SIGMA.2009.002

\begin{abstract}
Via a Cole-Hopf transformation, the multicomponent linear heat hierarchy leads to a multicomponent Burgers hierarchy. We show in particular that any solution of the latter also solves a corresponding multicomponent (potential) KP hierarchy. A generalization of the Cole-Hopf transformation leads to a more general relation between the multicomponent linear heat hierarchy and the multicomponent KP hierarchy. From this results a construction of exact solutions of the latter via a matrix linear system.
\end{abstract}

Key words: multicomponent KP hierarchy; Burgers hierarchy; Cole-Hopf transformation; Davey-Stewartson equation; Riccati equation; dromion

2000 Mathematics Subject Classification: 37K10; 35Q53

\section{Introduction}

The well-known Cole-Hopf transformation $\phi=\psi_{x} \psi^{-1}$ translates the nonlinear Burgers equation into the linear heat equation $[1,2,3]$. This extends to a corresponding relation between the Burgers hierarchy and the linear heat hierarchy, and moreover to their matrix generalizations. The Cole-Hopf transformation also generates solutions of the KP hierarchy from (invertible) solutions of the linear heat hierarchy $[4,5,6]$, and this extends to the corresponding matrix hierarchies $[7,8,9]$. There is also a generalization of the Cole-Hopf transformation that produces a solution of the (scalar or matrix) KP hierarchy from two solutions of the matrix linear heat hierarchy, connected by an additional relation [7, 8]. Furthermore, this includes a construction of (matrix) KP solutions from a matrix linear system (see also [10, 11]), which may be regarded as a finite-dimensional version of the Sato theory $[12,13]$. In this work we extend these results to the multicomponent case. Admittedly, this is not a very difficult task, on the basis of our previous results [7, 11]. We also take this opportunity, however, to present these results in a concise form and to add some relevant remarks and examples.

The usual multicomponent KP (mcKP) hierarchy contains subhierarchies that generalize the matrix KP hierarchy by modifying it with some constant matrix $B$ different from the identity matrix. We are particularly interested in such matrix hierarchies different from the ordinary matrix KP hierarchy. The Davey-Stewartson equation [14, 15], a two-dimensional nonlinear Schrödinger equation that appeared as a shallow-water limit of the Benney-Roskes

${ }^{\star}$ This paper is a contribution to the Proceedings of the XVIIth International Colloquium on Integrable Systems and Quantum Symmetries (June 19-22, 2008, Prague, Czech Republic). The full collection is available at http://www.emis.de/journals/SIGMA/ISQS2008.html 
equation $[16,17]$, emerges from such a modified matrix hierarchy (see Section 3). It is of course well-known to arise from the two-component KP hierarchy.

Section 2 recalls the Cole-Hopf transformation for a multicomponent Burgers (mcBurgers) hierarchy and Section 3 reveals relations between the mcBurgers and the mcKP hierarchy. Section 4 presents the abovementioned kind of generalization of the Cole-Hopf transformation which determines solutions of the mcKP hierarchy. From this derives a fairly simple method to construct mcKP solutions. This is the subject of Section 5, which also presents some examples. All this is closely related to a multicomponent version of a matrix Riccati hierarchy, as briefly explained in Section 6. Section 7 contains some conclusions.

In the following, let $\mathcal{A}$ be an associative (and typically noncommutative) algebra over the complex (or real) numbers, with identity element $I$ and supplied with a structure that allows to define derivatives with respect to real variables. Let $\mathcal{B}$ be a finite set of mutually commuting elements of $\mathcal{A}$. Although this assumption is sufficient to establish the results in this work, further assumptions should be placed on $\mathcal{B}$ in particular in order to diminish redundancy, see the remark at the end of Section 2. With each $B \in \mathcal{B}$ we associate a sequence of (real or complex) variables $\mathbf{t}_{B}=\left(t_{B, 1}, \ldots, t_{B, n}, \ldots\right)$. Furthermore, for a function $f$ of $\left\{\mathbf{t}_{B}\right\}_{B \in \mathcal{B}}$ we define a Miwa shift with respect to $B \in \mathcal{B}$ by $f_{[\lambda]_{B}}\left(\ldots, \mathbf{t}_{B}, \ldots\right)=f\left(\ldots, \mathbf{t}_{B}+[\lambda], \ldots\right)$, where $[\lambda]=(\lambda, \lambda / 2, \lambda / 3, \ldots)$ and $\lambda$ is an indeterminate. $f_{t_{n}}$ denotes the partial derivative of $f$ with respect to the variable $t_{n}$.

\section{Cole-Hopf transformation for a multicomponent Burgers hierarchy}

Let us consider the multicomponent linear heat hierarchy

$$
\psi_{t_{B, n}}=B^{n} \partial^{n}(\psi) \quad \forall B \in \mathcal{B}, \quad n=1,2, \ldots
$$

where $\partial=\partial_{x}$ is the operator of partial differentiation with respect to a variable $x$, and $\psi$ has values in $\mathcal{A}$. Since $\psi_{t_{B, 1}}=B \psi_{x}$, this implies the ordinary linear heat hierarchy $\psi_{t_{B, n}}=\partial_{t_{B, 1}}^{n}(\psi)$. Any two flows (2.1) commute as a consequence of our assumptions for $\mathcal{B}$ (the elements do not depend on $\left\{\mathbf{t}_{B}\right\}_{B \in \mathcal{B}}$ and commute with each other). A functional representation ${ }^{1}$ of (2.1) is given by

$$
\lambda^{-1}\left(\psi-\psi_{-[\lambda]_{B}}\right)=B \psi_{x} \quad \forall B \in \mathcal{B} .
$$

Proposition 1. If $\psi$ is an invertible solution of the above multicomponent linear heat hierarchy, then

$$
\phi=\psi_{x} \psi^{-1}
$$

solves the multicomponent Burgers (mcBurgers) hierarchy associated with $\mathcal{B}$, given by the functional representation

$$
\Omega_{B}(\phi, \lambda)=0 \quad \forall B \in \mathcal{B},
$$

where

$$
\Omega_{B}(\phi, \lambda):=\lambda^{-1}\left(\phi-\phi_{-[\lambda]_{B}}\right)-\left(B \phi-\phi_{-[\lambda]_{B}} B\right) \phi-B \phi_{x}
$$

\footnotetext{
${ }^{1}$ We use this (not quite satisfactory) term for an equation that generates a sequence of equations by expansion in powers of an indeterminate.
} 
Proof. We have to consider the following system,

$$
\psi_{x}=\phi \psi, \quad \psi_{-[\lambda]_{B}}=(I-\lambda B \phi) \psi \quad \forall B \in \mathcal{B} .
$$

The integrability condition $\left(\psi_{x}\right)_{-[\lambda]_{B}}=\left(\psi_{-[\lambda]_{B}}\right)_{x}$ yields $(2.4)$. The further integrability condition $\left(\psi_{-[\lambda]_{B_{1}}}\right)_{-[\mu]_{B_{2}}}=\left(\psi_{-[\mu]_{B_{2}}}\right)_{-[\lambda]_{B_{1}}}$ is

$$
B_{2}\left(\lambda^{-1}\left(\phi-\phi_{-[\lambda]_{B_{1}}}\right)+\phi_{-[\lambda]_{B_{1}}} B_{1} \phi\right)=B_{1}\left(\mu^{-1}\left(\phi-\phi_{-[\mu]_{B_{2}}}\right)+\phi_{-[\mu]_{B_{2}}} B_{2} \phi\right),
$$

which is satisfied as a consequence of $(2.4)$ and $\left[B_{1}, B_{2}\right]=0$.

(2.3) is a Cole-Hopf transformation. The first equation that results from (2.4) is

$$
\phi_{t_{B, 1}}=B \phi_{x}+[B, \phi] \phi,
$$

which has been called $C$-integrable $N$-wave equation in [9]. If $B=I$, this reduces to $t_{I, 1}=x$. But it is a nontrivial nonlinear equation if $\phi$ does not commute with $B$. The next equation that results from (2.4) is the (noncommutative) Burgers equation

$$
\phi_{t_{B, 2}}=\phi_{t_{B, 1} t_{B, 1}}+2 \phi_{t_{B, 1}} B \phi
$$

Remark 1. In order to avoid redundancy and to maximally extend the hierarchies, further conditions have to be imposed on $\mathcal{B}$. In particular, the elements of $\mathcal{B}$ should be linearly independent, since linear combinations correspond to linear combinations of hierarchy equations. But since products of elements of $\mathcal{B}$ also generate (independent or redundant) commuting flows, the problem is more subtle. If the algebra is semisimple, it admits a maximal set of commuting mutually annihilating idempotents $E_{a}, a=1, \ldots, N$, hence $E_{a} E_{b}=\delta_{a, b} E_{a}$ for all $a, b=1, \ldots, N$. Then $\mathcal{B}=\left\{E_{a}\right\}_{k=1}^{N}$ is an optimal choice. In fact, in the following we do not really address those cases of (non-semisimple) algebras where such a choice does not exist. Rather it turns out that

some more flexibility in the choice of $\mathcal{B}$ can be used to obtain certain integrable systems within this framework in a more direct way, see Section 3.

\section{Multicomponent KP and relations with the multicomponent Burgers hierarchy}

For $B \in \mathcal{B}$ let

$$
\mathcal{E}_{B}(\lambda):=I-\lambda\left(\omega_{B}(\lambda)+B \partial\right) .
$$

The "discrete" zero curvature condition

$$
\mathcal{E}_{B_{1}}(\lambda)_{-[\mu]_{B_{2}}} \mathcal{E}_{B_{2}}(\mu)=\mathcal{E}_{B_{2}}(\mu)_{-[\lambda]_{B_{1}}} \mathcal{E}_{B_{1}}(\lambda)
$$

then leads to the two equations

$$
\begin{aligned}
& \lambda^{-1}\left(\omega_{B_{2}}(\mu)-\omega_{B_{2}}(\mu)_{-[\lambda]_{B_{1}}}\right)+\omega_{B_{2}}(\mu)_{-[\lambda]_{B_{1}}} \omega_{B_{1}}(\lambda)+B_{2} \omega_{B_{1}}(\lambda)_{x} \\
& \quad=\mu^{-1}\left(\omega_{B_{1}}(\lambda)-\omega_{B_{1}}(\lambda)_{-[\mu]_{B_{2}}}\right)+\omega_{B_{1}}(\lambda)_{-[\mu]_{B_{2}}} \omega_{B_{2}}(\mu)+B_{1} \omega_{B_{2}}(\mu)_{x}
\end{aligned}
$$

and

$$
B_{2} \omega_{B_{1}}(\lambda)-\omega_{B_{1}}(\lambda)_{-[\mu]_{B_{2}}} B_{2}=B_{1} \omega_{B_{2}}(\mu)-\omega_{B_{2}}(\mu)_{-[\lambda]_{B_{1}}} B_{1} .
$$

The last equation is solved by

$$
\omega_{B}(\lambda)=B \phi-\phi_{-[\lambda]_{B}} B,
$$


and the first equation can then be written in terms of (2.5) as

$$
B_{2} \Omega_{B_{1}}(\phi, \lambda)-\Omega_{B_{1}}(\phi, \lambda)_{-[\mu]_{B_{2}}} B_{2}=B_{1} \Omega_{B_{2}}(\phi, \mu)-\Omega_{B_{2}}(\phi, \mu)_{-[\lambda]_{B_{1}}} B_{1} \quad \forall B_{1}, B_{2} \in \mathcal{B} .
$$

We take this as our defining equations of the (more precisely, potential) multicomponent KP (mcKP) hierarchy associated with $\mathcal{B} .^{2}$

Remark 2. Choosing $B_{1}=B_{2}=B$ in (3.2) and summing the resulting equation three times with cyclically permuted indeterminates, leads to

$$
\sum_{i, j, k=1}^{3} \epsilon_{i j k}\left(\lambda_{i}^{-1}\left(\phi-\phi_{-\left[\lambda_{i}\right]_{B}}\right)+\phi_{-\left[\lambda_{i}\right]_{B}} B \phi\right)_{-\left[\lambda_{j}\right]_{B}} B=0,
$$

which is a special case of the functional form of the mcKP hierarchy in [27].

Let us take a closer look at $(3.2)$ with $B_{1}=B_{2}=B$. Its $\lambda$-independent part is

$$
B \Omega_{B}(\phi, 0)-\Omega_{B}(\phi, 0)_{-[\mu]_{B}} B=\left[B, \Omega_{B}(\phi, \mu)\right]
$$

where $\Omega_{B}(\phi, 0)=\phi_{t_{B, 1}}-[B, \phi] \phi-B \phi_{x}$. To first order in $\mu$ this gives

$$
B \phi_{t_{B, 1} x} B-\frac{1}{2}\left\{B, \phi_{t_{B, 1} t_{B, 1}}\right\}+\frac{1}{2}\left[B, \phi_{t_{B, 2}}\right]=B \phi_{t_{B, 1}}[B, \phi]-[B, \phi] \phi_{t_{B, 1}} B .
$$

(3.2) is the integrability condition of

$$
\Omega_{B}(\phi, \lambda)=B \theta-\theta_{-[\lambda]_{B}} B \quad \forall B \in \mathcal{B},
$$

with a new dependent variable $\theta$. (3.4) represents the mcKP hierarchy in terms of two dependent variables. By comparison with (2.4), this has the form of an inhomogeneous mcBurgers hierarchy. The following is an immediate consequence ${ }^{3}$.

Proposition 2. Any solution of the mcBurgers hierarchy also solves the mcKP hierarchy.

Proof. (3.4) becomes (2.4) if $\theta=0$.

The representation (3.4) of the mcKP hierarchy has the advantage that each equation only involves a single element from the set $\mathcal{B}$. To order $\lambda^{0},(3.4)$ yields

$$
\phi_{t_{B, 1}}-B \phi_{x}-[B, \phi] \phi=[B, \theta] .
$$

If $B=I$, then (3.5) reduces to $\phi_{t_{B, 1}}=\phi_{x}$. Otherwise this is a non-trivial nonlinear equation. Subtracting (3.5) from (3.4), leads to

$$
\lambda^{-1}\left(\phi-\phi_{-[\lambda]_{B}}\right)-\phi_{t_{B, 1}}-\left(\phi-\phi_{-[\lambda]_{B}}\right) B \phi=\left(\theta-\theta_{-[\lambda]_{B}}\right) B .
$$

The two equations (3.5) and (3.6) are equivalent to (3.4). (3.6) does not involve derivatives with respect to $x$. For fixed $B \in \mathcal{B}$, it represents the KP hierarchy in $\mathcal{A}$ [7], with product modified by $B$.

\footnotetext{
${ }^{2}$ See also e.g. $[18,19,20,21,22,6,23,24,25,26,27]$ for different formulations of such a multicomponent KP hierarchy. We should also mention that the conditions imposed on the set $\mathcal{B}$ can be relaxed while keeping the hierarchy property, see [26] for example.

${ }^{3}$ It was first noted in [4] that any solution of the first two equations of the (scalar) Burgers hierarchy also solves the (scalar potential) KP equation. In [28, 29] the (first two) Burgers hierarchy equations have been recovered via a symmetry constraint from the KP hierarchy and its linear system.
} 
Proposition 3. For any $B \in \mathcal{B}$, as a consequence of the mcKP hierarchy (3.4), B $\phi$ and also $\phi B$ solves the ordinary (noncommutative) KP hierarchy.

Proof. This is an immediate consequence of (3.6).

To first order in $\lambda^{-1},(3.6)$ yields

$$
\frac{1}{2}\left(\phi_{t_{B, 2}}-\phi_{t_{B, 1} t_{B, 1}}\right)-\phi_{t_{B, 1}} B \phi=\theta_{t_{B, 1}} B .
$$

Differentiating (3.5) with respect to $t_{B, 1}$, and multiplying it by $B$ from the right, we can use the last equation to eliminate $\theta$. In this way we recover (3.3). As shown in Example 2 below, (3.3) generalizes the Davey-Stewartson (DS) equation $[16,14,15,17]$. Eliminating $\theta$ from (3.6) by use of (3.5) thus leads to a (generalized) DS hierarchy.

Example 1. Let $B=\sigma$, where $\sigma^{2}=I$. Decomposing $\phi$ as

$$
\phi=D+U, \quad \text { where } \quad D:=\frac{1}{2}(\phi+\sigma \phi \sigma), \quad U:=\frac{1}{2}(\phi-\sigma \phi \sigma),
$$

(3.3) splits into the two equations ${ }^{4}$

$$
\begin{aligned}
& D_{t_{1}} \sigma-D_{x}-2 U^{2}=0, \\
& U_{t_{2}} \sigma+U_{x t_{1}}+2\left\{U, D_{t_{1}}\right\}=0,
\end{aligned}
$$

where we write $t_{1}, t_{2}$ instead of $t_{B, 1}$ and $t_{B, 2}$. Let now $\mathcal{A}$ be the algebra of $2 \times 2$ matrices over $\mathbb{C}$, and $\sigma=\operatorname{diag}(1,-1)$. Then $D$ and $U$ are diagonal and off-diagonal parts of $\phi$, respectively. Writing

$$
U=\left(\begin{array}{cc}
0 & u \\
v & 0
\end{array}\right), \quad \text { hence } \quad u:=\phi_{1,2}, \quad v:=\phi_{2,1},
$$

and introducing

$$
s:=\operatorname{tr}(\phi)=\phi_{1,1}+\phi_{2,2}, \quad r:=\phi_{1,1}-\phi_{2,2},
$$

we obtain the system

$$
u_{t_{2}}-u_{x t_{1}}-2 u s_{t_{1}}=0, \quad v_{t_{2}}+v_{x t_{1}}+2 v s_{t_{1}}=0,
$$

and $s_{t_{1}}=r_{x}, s_{x}=r_{t_{1}}-4 u v$. The integrability conditions of the latter two equations are

$$
s_{t_{1} t_{1}}-s_{x x}=4(u v)_{x}, \quad r_{t_{1} t_{1}}-r_{x x}=4(u v)_{t_{1}} .
$$

The two equations (3.10) together with the first of (3.11) constitute a fairly simple system of three nonlinear coupled equations, where all variables can be taken to be real. Allowing complex dependent and independent variables, after a complex transformation the system for the dependent variables $u, v, s$ can be further reduced to the DS equation, see the next example. Of course, the above system (3.10) and (3.11) can also be derived from the usual two-component KP hierarchy (see e.g. [27]), and the transformation to DS is well-known.

Setting $v=0$, we obtain from (3.10) and (3.11) the following linear equations ${ }^{5}$,

$$
u_{t_{2}}-u_{x t_{1}}-2 \varphi u=0, \quad \varphi_{t_{1} t_{1}}-\varphi_{x x}=0,
$$

where $\varphi:=s_{t_{1}}=\operatorname{tr}(\phi)_{t_{1}}$. This is probably the simplest system that possesses dromion solutions, as observed in Example 3 in Section 5.

\footnotetext{
${ }^{4} \mathrm{~A}$ constant (with respect to $t_{1}$ ) of integration has been set to zero in order to obtain (3.7). The latter equation can be obtained more directly as the diagonal part of (3.5).

${ }^{5}$ If $u$ does not depend on $t_{2}$, then the first equation is part of a Lax pair for the Nizhnik-Novikov-Veselov equation.
} 
Example 2. We continue with the previous example and perform a transformation to the DS equation by first allowing the dependent variables to live in a noncommutative algebra. In this way we obtain a certain noncommutative generalization of the DS equation (see also [30,31] for matrix DS versions). In terms of

$$
F=D_{t_{1}}+\beta U^{2},
$$

with $\beta \in \mathbb{C}$, (3.7) becomes

$$
F_{t_{1}} \sigma-F_{x}=-\beta\left(U^{2}\right)_{x}+\left(U^{2}\right)_{t_{1}}(2+\beta \sigma) .
$$

Differentiating this with respect to $x$ and with respect to $t_{1}$, respectively, and eliminating mixed derivatives of $F$ from the resulting two equations, yields

$$
F_{t_{1} t_{1}}-F_{x x}=-\beta\left(U^{2}\right)_{x x}+2\left(U^{2}\right)_{x t_{1}}+\left(U^{2}\right)_{t_{1} t_{1}}(2 \sigma+\beta) .
$$

Furthermore, (3.8) takes the form

$$
U_{t_{2}} \sigma+U_{x t_{1}}=-2\{U, F\}+4 \beta U^{3} .
$$

Let now $\mathcal{A}$ be the algebra of $2 \times 2$ matrices over some unital associative algebra with unit denoted by 1 , and $\sigma=\operatorname{diag}(1,-1)$. Using (3.9) and writing

$$
F=\operatorname{diag}(f, g),
$$

(3.13) and (3.14) result in the following equations,

$$
\begin{aligned}
& u_{t_{2}}-u_{x t_{1}}=2(f u+u g)-4 \beta u v u, \\
& v_{t_{2}}+v_{x t_{1}}=-2(g v+v f)+4 \beta v u v, \\
& f_{t_{1} t_{1}}-f_{x x}=-\beta(u v)_{x x}+2(u v)_{x t_{1}}+(2+\beta)(u v)_{t_{1} t_{1}}, \\
& g_{t_{1} t_{1}}-g_{x x}=-\beta(v u)_{x x}+2(v u)_{x t_{1}}-(2-\beta)(v u)_{t_{1} t_{1}} .
\end{aligned}
$$

In terms of the new variables

$$
y=\frac{1+\mathrm{i}}{\kappa \sqrt{2}}\left(x+\mathrm{i} t_{1}\right), \quad z=\frac{1-\mathrm{i}}{\sqrt{2}}\left(x-\mathrm{i} t_{1}\right),
$$

with a constant $\kappa \neq 0$, and with the choice $\beta=\mathrm{i}$, this becomes

$$
\begin{aligned}
& u_{t_{2}}+u_{z z}+\frac{1}{\kappa^{2}} u_{y y}=2(f u+u g)-4 \mathrm{i} u v u, \\
& -v_{t_{2}}+v_{z z}+\frac{1}{\kappa^{2}} v_{y y}=2(g v+v f)+4 \mathrm{i} v u v, \\
& f_{z z}-\frac{1}{\kappa^{2}} f_{y y}=(1+2 \mathrm{i})(u v)_{z z}-\frac{1}{\kappa^{2}}(u v)_{y y}-\frac{2 \mathrm{i}}{\kappa}(u v)_{y z}, \\
& g_{z z}-\frac{1}{\kappa^{2}} g_{y y}=(-1+2 \mathrm{i})(v u)_{z z}+\frac{1}{\kappa^{2}}(v u)_{y y}+\frac{2 \mathrm{i}}{\kappa}(v u)_{y z} .
\end{aligned}
$$

If the dependent variables take their values in a commutative algebra, then we obtain

$$
\begin{aligned}
& u_{t_{2}}+u_{z z}+\frac{1}{\kappa^{2}} u_{y y}=2 \rho u-4 \mathrm{i} u^{2} v, \\
& -v_{t_{2}}+v_{z z}+\frac{1}{\kappa^{2}} v_{y y}=2 \rho v+4 \mathrm{i} v^{2} u \\
& \rho_{z z}-\frac{1}{\kappa^{2}} \rho_{y y}=4 \mathrm{i}(u v)_{z z},
\end{aligned}
$$


where

$$
\rho:=f+g=\varphi+2 \mathrm{i} u v
$$

(with $\left.\varphi=\operatorname{tr}(\phi)_{t_{1}}\right)$. Setting $t_{2}=-\mathrm{i} t$ and

$$
v=\mathrm{i} \epsilon \bar{u} \quad \text { with } \quad \epsilon= \pm 1
$$

and the complex conjugate $\bar{u}$ of $u$, one recovers the DS system

$$
\mathrm{i} u_{t}+u_{z z}+\frac{1}{\kappa^{2}} u_{y y}=2 \rho u+4 \epsilon|u|^{2} u, \quad \rho_{z z}-\frac{1}{\kappa^{2}} \rho_{y y}=-4 \epsilon\left(|u|^{2}\right)_{z z},
$$

where now $y, z, t, \kappa^{2}, \rho$ are taken to be real. Clearly, this system is more quickly obtained from (3.10) and (3.11) by application of the transformation of independent variables given by (3.15) and $t_{2}=-\mathrm{i} t$, and the transformation (3.17) of dependent variables. But on our way we obtained the system (3.16) which (with $t_{2}=-\mathrm{i} t$ ) may be of interest as a noncommutative version of the DS equation (see [30,31] for alternatives).

We conclude that the DS equation (and a corresponding hierarchy) is obtained from a matrix KP hierarchy, but the latter has to be generalized by introduction of a matrix $B$ different from the unit matrix. Of course, this hierarchy is embedded in the usual two-component KP hierarchy.

\subsection{The associated Sato-Wilson system and its translation into a Burgers hierarchy}

(3.1) is the integrability condition of the linear system

$$
\tilde{\psi}_{-[\lambda]_{B}}=\mathcal{E}_{B}(\lambda) \tilde{\psi} \quad \forall B \in \mathcal{B} .
$$

If $W$ is an invertible solution of

$$
\mathcal{E}_{B}(\lambda) W=W_{-[\lambda]_{B}} \mathcal{E}_{0, B}(\lambda), \quad \mathcal{E}_{0, B}(\lambda):=I-\lambda B \partial,
$$

then the linear system is mapped to $\psi_{-[\lambda]_{B}}=\mathcal{E}_{0, B}(\lambda) \psi$ where $\psi:=W^{-1} \tilde{\psi}$. The latter is the linear heat hierarchy (2.2). The ansatz

$$
W=I+\sum_{n \geq 1} w_{n} \partial^{-n}
$$

inserted into the functional form (3.19) of the Sato-Wilson equations, leads to

$$
\lambda^{-1}\left(w_{n}-w_{n,-[\lambda]_{B}}\right)-B w_{n, x}-\left(B \phi-\phi_{-[\lambda]_{B}} B\right) w_{n}=B w_{n+1}-w_{n+1,-[\lambda]_{B}} B,
$$

where $n=0,1, \ldots$ and $w_{0}=I$. From the $n=0$ equation we get $w_{1}=-\phi$. In terms of $\theta:=-w_{2}$, the $n=1$ equation turns out to be the functional form (3.4) of the mcKP hierarchy. Let us introduce

$$
\Phi:=\Lambda^{\top}+e_{1}\left(\phi,-w_{2},-w_{3}, \ldots\right),
$$

where $e_{1}^{\top}=(I, 0, \ldots)$, and

$$
\Lambda:=\left(\begin{array}{ccccc}
0 & I & 0 & 0 & \ldots \\
0 & 0 & I & 0 & \ldots \\
\vdots & \vdots & \ddots & \ddots & \ddots
\end{array}\right)
$$


is the shift operator matrix with transpose $\Lambda^{\top}$. Then (3.20) can be expressed as the following mcBurgers hierarchy,

$$
\Omega_{B}(\Phi, \lambda) \equiv \lambda^{-1}\left(\Phi-\Phi_{-[\lambda]_{B}}\right)-\left(B \Phi-\Phi_{-[\lambda]_{B}} B\right) \Phi-B \Phi_{x}=0 \quad \forall B \in \mathcal{B} .
$$

The linear system of the mcKP hierarchy has thus been reformulated as an mcBurgers hierarchy with an infinite matrix variable of a special form.

We note that (3.21) has the form of a companion matrix, a block of a Frobenius normal form matrix. This makes contact with recent work in [9], where several integrable systems were recovered from equations of a Burgers hierarchy with dependent variable of Frobenius normal form. All these systems are known to arise as reductions of the mcKP hierarchy.

As a consequence of (3.23) with $\Phi$ of the form (3.21), it follows that $\phi=e_{1}^{\top} \Phi e_{1}$ solves the mcKP hierarchy in $\mathcal{A}$.

In the following section, we shall see that $\Phi$ of the form (3.21) results, as a particular case, from a quite general result about solutions of a somewhat generalized mcKP hierarchy.

\section{Generalization of the Cole-Hopf transformation}

We generalize (3.4) to

$$
\Omega_{B, Q}(\Phi, \lambda)=B \Theta-\Theta_{-[\lambda]_{B}} B \quad \forall B \in \mathcal{B},
$$

where

$$
\Omega_{B, Q}(\Phi, \lambda):=\lambda^{-1}\left(\Phi-\Phi_{-[\lambda]_{B}}\right)-\left(B \Phi-\Phi_{-[\lambda]_{B}} B\right) Q \Phi-B \Phi_{x},
$$

with a constant object $Q$. We assume that the constituents are elements of linear spaces and that the products are defined. (4.1) determines a generalization of the mcKP hierarchy, since nonlinear terms now involve $Q$ (which modifies the product). The following generalizes a theorem in [7] (see also [8]).

Theorem 1. Let $X, Y$ be solutions of the multicomponent linear heat hierarchy, i.e.

$$
\lambda^{-1}\left(X-X_{-[\lambda]_{B}}\right)=B X_{x}, \quad \lambda^{-1}\left(Y-Y_{-[\lambda]_{B}}\right)=B Y_{x},
$$

for all $B \in \mathcal{B}$, and furthermore

$$
X_{x}=R X+Q Y,
$$

with constant objects $R, Q$. If $X$ is invertible and if all $B \in \mathcal{B}$ commute with $R$, then

$$
\Phi=Y X^{-1}
$$

solves the $m c K P_{Q}$ hierarchy (4.1) with $\Theta=\Phi R$.

Proof. Using (4.5) in the definition (4.2), we have

$$
\begin{aligned}
\Omega_{B, Q}(\Phi, \lambda)= & \left(B \Phi-\Phi_{-[\lambda]_{B}} B\right)\left(X_{x}-Q Y\right) X^{-1}+\left(\lambda^{-1}\left(Y-Y_{-[\lambda]_{B}}\right)-B Y_{x}\right) X^{-1} \\
& -\Phi_{-[\lambda]_{B}}\left(\lambda^{-1}\left(X-X_{-[\lambda]_{B}}\right)-B X_{x}\right) X^{-1}
\end{aligned}
$$

which reduces to

$$
\Omega_{B, Q}(\Phi, \lambda)=\left(B \Phi-\Phi_{-[\lambda]_{B}} B\right) R
$$

as a consequence of (4.3) and (4.4). Since $[B, R]=0$, this takes the form (4.1) with $\Theta=\Phi R$. 
Now we set up the stage for applications of the theorem. Let $\mathbb{A}(M, N):=\operatorname{Mat}(M \times N, \mathbb{C}) \otimes \mathcal{A}$, where $\operatorname{Mat}(M \times N, \mathbb{C})$ is the space of complex $M \times N$ matrices. Let $\Phi, \Theta, Y$ take values in $\mathbb{A}(M, N)$, and $X$ in $\mathbb{A}(N, N)$. Furthermore, let $Q \in \mathbb{A}(N, M)$ and $R \in \mathbb{A}(N, N)$ commute with all $B \in \mathcal{B}$.

If $Q$ has rank one over $\mathcal{A}$, in the sense that $Q=V U^{\top}$ with constant vectors $U$, $V$, with entries in $\mathcal{A}$, and if $U$ and $V$ commute with all $B \in \mathcal{B}$, then $\phi=U^{\top} \Phi V$ solves the mcKP hierarchy in $\mathcal{A}$, provided that $\Phi$ solves (4.1). In this way, any solution $X, Y$ of the linear equations formulated in the above theorem generates an $\mathcal{A}$-valued solution $\phi$ of the mcKP hierarchy (3.2).

Choosing $M=N,(4.5)$ is a Cole-Hopf transformation if $Y=X_{x}$. Then (4.4) becomes $(I-Q) X_{x}=R X$. Let $\mathbf{e}_{k}$ denote the $N$-component vector with all entries zero except for the identity element in the $k$ th row.

a) Setting $Q=\mathbf{e}_{N} \mathbf{e}_{N}^{\top}$ and $R=\sum_{k=1}^{N-1} \mathbf{e}_{k} \mathbf{e}_{k+1}^{\top}$ (which is the left shift operator: $R \mathbf{e}_{k}=\mathbf{e}_{k-1}$, $k=2, \ldots, N$, and $R \mathbf{e}_{1}=0$ ), one finds that (4.4) restricts $X$ to the form of a Wronski matrix (see also [8]),

$$
X=\left(\begin{array}{cccc}
X^{(1)} & X^{(2)} & \cdots & X^{(N)} \\
\partial\left(X^{(1)}\right) & \partial\left(X^{(2)}\right) & \cdots & \partial\left(X^{(N)}\right) \\
\vdots & \vdots & \ddots & \vdots \\
\partial^{N-1}\left(X^{(1)}\right) & \partial^{N-1}\left(X^{(2)}\right) & \cdots & \partial^{N-1}\left(X^{(N)}\right)
\end{array}\right)
$$

$X^{(1)}, X^{(2)}, \ldots, X^{(N)}$ are independent functions with values in $\mathcal{A}$. The remaining assumption (4.3) in the theorem requires them to be solutions of the multicomponent heat hierarchy.

b) Let $R=\Lambda^{\top}$ with the infinite shift operator matrix (3.22), and $Q=\mathbf{e}_{1} \mathbf{e}_{1}^{\top}$. Then (4.4) says that $X$ has to be a pseudo-Wronski matrix

$$
X=\left(\begin{array}{cccc}
X^{(1)} & X^{(2)} & X^{(3)} & \cdots \\
\partial^{-1} X^{(1)} & \partial^{-1} X^{(2)} & \partial^{-1} X^{(3)} & \cdots \\
\partial^{-2} X^{(1)} & \partial^{-2} X^{(2)} & \partial^{-2} X^{(3)} & \cdots \\
\vdots & \vdots & \vdots & \ddots
\end{array}\right)
$$

where $\partial^{-1}$ is the formal inverse of $\partial$. This structure appeared in [9] (equations (14) and (50) therein). (4.3) demands that $X^{(1)}, X^{(2)}, \ldots$ solve the multicomponent heat hierarchy. With (4.6), $\Phi=X_{x} X^{-1}$ has the form (3.21) and hence determines a solution of (3.23).

\section{Solutions of the multicomponent KP hierarchy from a matrix linear system}

In order to derive some classes of mcKP solutions via theorem 1 more explicitly, in the framework specified in Section 4 (after the theorem) we extend (4.4) to

$$
Z_{x}=H Z,
$$

where

$$
Z=\left(\begin{array}{c}
X \\
Y
\end{array}\right), \quad H=\left(\begin{array}{cc}
R & Q \\
S & L
\end{array}\right),
$$

with new constant objects $L \in \mathbb{A}(M, M)$ and $S \in \mathbb{A}(M, N)$ that commute with all $B \in \mathcal{B}$. The two equations (4.3) then combine to

$$
\lambda^{-1}\left(Z-Z_{-[\lambda]_{B}}\right)=B Z_{x} \quad \forall B \in \mathcal{B} .
$$


Taking (5.1) into account, this is equivalent to

$$
Z_{t_{B, n}}=(B H)^{n} Z \quad \forall B \in \mathcal{B}, \quad n=1,2, \ldots
$$

Note that $B$ and $H$ commute as a consequence of our assumptions. With a suitable choice of the algebra $\mathcal{A}$, the solution of the matrix linear system (5.1), (5.2) is given by

$$
Z(x, \mathbf{t})=e^{\xi(x, \mathbf{t} ; H, B)} Z_{0}
$$

where

$$
\xi(x, \mathbf{t} ; H, B):=x H+\sum_{B \in \mathcal{B}} \sum_{n=1}^{\infty} t_{B, n}(H B)^{n},
$$

and $\mathbf{t}$ stands for $\left\{\mathbf{t}_{B}\right\}_{B \in \mathcal{B}}$. Decomposing $Z$ into $X$ and $Y$, theorem 1 implies that $\Phi=Y X^{-1}$ solves the $\operatorname{mcKP}_{Q}$ hierarchy (4.1). Furthermore, if $\operatorname{rank}(Q)=1$ over $\mathcal{A}$, hence $Q=V U^{\top}$ with constant vectors $U$ and $V$, then the $\mathcal{A}$-valued variable

$$
\phi=U^{\top} \Phi V
$$

solves the corresponding mcKP hierarchy.

The exponential in (5.3) can be computed explicitly if additional assumptions are made concerning the form of $H$ (see [11], in particular). Then $\Phi$ is obtained via (4.5).

Case 1. Let $S=0$ and

$$
Q=R K-K L
$$

with a constant $N \times M$ matrix $K($ over $\mathcal{A}$ ) that commutes with all $B \in \mathcal{B}$. Then we obtain

$$
\Phi=e^{\xi(x, \mathbf{t} ; L, B)} C e^{-\xi(x, \mathbf{t} ; R, B)}\left(I_{N}-K e^{\xi(x, \mathbf{t} ; L, B)} C e^{-\xi(x, \mathbf{t} ; R, B)}\right)^{-1},
$$

where $I_{N}$ is the $N \times N$ unit matrix over $\mathcal{A}$ (so that the diagonal entries are the identity $I$ in $\mathcal{A}$ ), and $C$ is an arbitrary constant $M \times N$ matrix (with entries in $\mathcal{A}$ ). $\Phi$ solves the $\operatorname{mcKP}_{Q}$ hierarchy (associated with $\mathcal{B}$ ), with $Q$ given by (5.4). ${ }^{6}$ If moreover $Q=V U^{\top}$ with vectors $U, V$ that commute with all $B \in \mathcal{B}$, then $\phi=U^{\top} \Phi V$ solves the mcKP hierarchy in $\mathcal{A}$. Of course, it remains to solve the rank one condition (over $\mathcal{A}$ )

$$
R K-K L=V U^{\top}
$$

If $M=N$ and if $C$ is invertible, then (5.5) simplifies to

$$
\Phi=\left(e^{\xi(x, \mathbf{t} ; R, B)} C^{-1} e^{-\xi(x, \mathbf{t} ; L, B)}-K\right)^{-1},
$$

which remains a solution if we replace $C^{-1}$ by an arbitrary constant $N \times N$ matrix $\tilde{C}$ (with entries in $\mathcal{A}$ ).

Example 3. Choosing the components of the matrices $L, R$ as

$$
L_{i j}=p_{i} \delta_{i j} I, \quad R_{k l}=q_{k} \delta_{k l} I,
$$

\footnotetext{
${ }^{6}$ In particular, if $M=N$ and $Q=I_{N}$, then $\Phi$ (with $K, L, R$ solving $R K-K L=I_{N}$ ) is a solution of the $N \times N$ matrix (over $\mathcal{A}$ ) mcKP hierarchy.
} 
with constants $p_{i}, q_{k},(5.6)$ is solved by

$$
K_{k j}=\frac{1}{q_{k}-p_{j}} u_{k} v_{j}
$$

where $u_{k}$ and $v_{j}$ are the components of $U$ and $V$, respectively. We elaborate one of the simplest cases in some detail. Let us choose $\mathcal{A}$ as the algebra of $2 \times 2$ matrices over $\mathbb{C}, L=p I_{2}, R=q I_{2}$, with constants $p$ and $q, Q=I=I_{2}$, and $\mathcal{B}=\{B\}$ with $B=\operatorname{diag}(1,-1)$ (motivated by the examples in Section 3). Then we have $\xi(x, \mathbf{t} ; L, B)=\operatorname{diag}\left(\xi_{+}(x, \mathbf{t} ; p), \xi_{-}(x, \mathbf{t} ; p)\right)$ with

$$
\xi_{ \pm}(x, \mathbf{t} ; p):=p x+\sum_{n=1}^{\infty} p^{2 n} t_{2 n} \pm \sum_{n=0}^{\infty} p^{2 n+1} t_{2 n+1}
$$

(writing $t_{n}$ instead of $\left.t_{B, n}\right)$, and $\xi(x, \mathbf{t} ; R, B)$ is obtained by exchanging $p$ by $q$ in these expressions. Writing

$$
C=\left(\begin{array}{ll}
c_{1} & c_{2} \\
c_{3} & c_{4}
\end{array}\right)
$$

with constants $c_{i}$, we obtain (with $\left.U=V=I_{2}\right)^{7}$

$$
\phi(x, \mathbf{t})=\frac{1}{\mathcal{D}(x, \mathbf{t})}\left(\begin{array}{cc}
c_{1} e^{\xi_{+}(x, \mathbf{t} ; p)-\xi_{+}(x, \mathbf{t} ; q)}+f(x, \mathbf{t}) & c_{2} e^{\xi_{+}(x, \mathbf{t} ; p)-\xi_{-}(x, \mathbf{t} ; q)} \\
c_{3} e^{\xi_{-}(x, \mathbf{t} ; p)-\xi_{+}(x, \mathbf{t} ; q)} & c_{4} e^{\xi_{-}(x, \mathbf{t} ; p)-\xi_{-}(x, \mathbf{t} ; q)}+f(x, \mathbf{t})
\end{array}\right),
$$

where

$$
\begin{aligned}
& f(x, \mathbf{t}):=\frac{c_{1} c_{4}-c_{2} c_{3}}{p-q} e^{\xi_{+}(x, \mathbf{t} ; p)+\xi_{-}(x, \mathbf{t} ; p)-\xi_{+}(x, \mathbf{t} ; q)-\xi_{-}(x, \mathbf{t} ; q)}, \\
& \mathcal{D}(x, \mathbf{t}):=1+\frac{1}{p-q}\left(c_{1} e^{\xi_{+}(x, \mathbf{t} ; p)-\xi_{+}(x, \mathbf{t} ; q)}+c_{4} e^{\xi_{-}(x, \mathbf{t} ; p)-\xi_{-}(x, \mathbf{t} ; q)}+f(x, \mathbf{t})\right) .
\end{aligned}
$$

This is a solution of (3.3), with $B=\operatorname{diag}(1,-1)$, and its hierarchy, and its components thus provide us with the solution

$$
\begin{aligned}
u & =\frac{c_{2}}{\mathcal{D}} e^{\xi_{+}(x, \mathbf{t} ; p)-\xi_{-}(x, \mathbf{t} ; q)}, \quad v=\frac{c_{3}}{\mathcal{D}} e^{\xi_{-}(x, \mathbf{t} ; p)-\xi_{+}(x, \mathbf{t} ; q)}, \\
s & =\frac{1}{\mathcal{D}}\left(c_{1} e^{\xi_{+}(x, \mathbf{t} ; p)-\xi_{+}(x, \mathbf{t} ; q)}+c_{4} e^{\xi_{-}(x, \mathbf{t} ; p)-\xi_{-}(x, \mathbf{t} ; q)}+2 f\right)
\end{aligned}
$$

of the system (3.10), (3.11). $\phi$ is regular (for all t) in particular if all constants are real, $c_{1} c_{4}>c_{2} c_{3}$, and either $p>q, c_{1}>0, c_{4}>0$, or $p<q, c_{1}<0, c_{4}<0$. Fig. 1 presents a dromion ${ }^{8}$ solution within this family.

If $c_{3}=0$, we have $v=0$ and (5.12) determines a solution of the linear equations (3.12). ${ }^{9}$ An extremum of $u$ for a regular solution then moves (in "time" $t_{2}$ ) with constant amplitude along the curve given by

$$
x=-(p+q) t_{2}-\frac{1}{2(p-q)} \log \left(\frac{c_{1} c_{4}}{(p-q)^{2}}\right), \quad t_{1}=\frac{1}{p-q} \log \left(-\frac{p}{q} \sqrt{\frac{c_{4}}{c_{1}}}\right) .
$$

The last expression shows that, for a dromion solution, $p$ and $q$ must have opposite signs.

\footnotetext{
${ }^{7}$ Since $\xi_{ \pm}(x, \mathbf{t} ; p)-\xi_{ \pm}(x, \mathbf{t} ; q)=(p-q)\left(x \pm t_{1}\right)+\left(p^{2}-q^{2}\right) t_{2}+\cdots$, this solution becomes independent of $t_{2}$ (i.e. "static") if $p=-q$.

${ }^{8}$ The characteristic features of a dromion are its exponential localization and that it is accompanied by a field structure of intersecting line solitons. See [32, 33, 34, 35, 36, 37, 38, 39, 40, 41] for the Davey-Stewartson case, and especially [42] for an illuminating structural analysis and the appearance of dromions as solutions of other equations.

${ }^{9}$ In this case, $\mathcal{D}$ factorizes,

$$
\mathcal{D}=\left(1+\frac{c_{1}}{p-q} e^{\xi_{+}(x, \mathbf{t} ; p)-\xi_{+}(x, \mathbf{t} ; q)}\right)\left(1+\frac{c_{4}}{p-q} e^{\xi_{-}(x, \mathbf{t} ; p)-\xi_{-}(x, \mathbf{t} ; q)}\right) .
$$
}



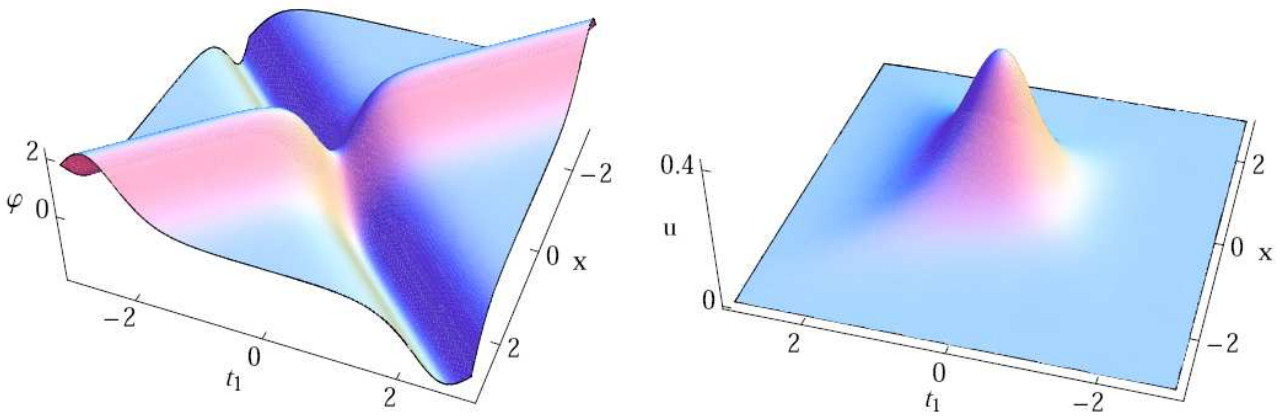

Figure 1. A dromion solution of (3.10) and (3.11) at $t_{2}=0$, given by (5.12) with $p=2, q=-1$, $c_{1}=c_{4}=1, c_{2}=1 / 2$ and $c_{3}=0$. The left plot shows $\varphi=s_{t_{1}}=\operatorname{tr}(\phi)_{t_{1}}$. As a consequence of $c_{3}=0$, we have $v=0$. Hence this is actually a solution of the linear equations (3.12) and $\varphi$ solves the linear wave equation. Regarding $t_{1}$ as an evolution parameter, the plot of $\varphi$ shows two colliding humps (with amplitudes having opposite signs) that annihilate at $t_{1}=0$. With $0 \neq c_{3}<2$, the plots remain qualitatively the same as long as $c_{3}$ is sufficiently far below the upper bound, and $v$ attains a shape similar to that of $u$.
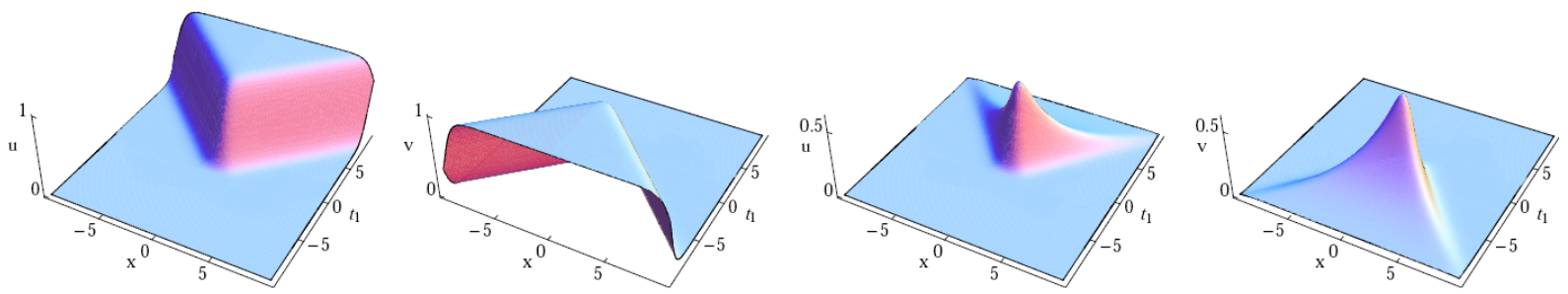

Figure 2. Solutions of (3.10) and (3.11) at $t_{2}=0$, given by (5.11) with $p=2, c_{1}=c_{4}=1, c_{2}=c_{3}=1 / 2$. The first two plots, where $q=0$, show kinks (see (5.13)). In the last two plots, where $q=-1 / 5$, these become exponentially localized structures.

For $q=0$ in (5.12), setting $t_{n}=0$ for $n>2$, we have

$$
\begin{aligned}
& u=c_{2} p^{2}\left(c_{1} p+c_{4} p e^{-2 p t_{1}}+e^{-p t_{1}}\left(p^{2} e^{-p\left(x+p t_{2}\right)}+\left(c_{1} c_{4}-c_{2} c_{3}\right) e^{p\left(x+p t_{2}\right)}\right)^{-1},\right. \\
& v=c_{3} p^{2}\left(c_{4} p+c_{1} p e^{2 p t_{1}}+e^{p t_{1}}\left(p^{2} e^{-p\left(x+p t_{2}\right)}+\left(c_{1} c_{4}-c_{2} c_{3}\right) e^{p\left(x+p t_{2}\right)}\right)^{-1}\right.
\end{aligned}
$$

If $c_{1} c_{4}>c_{2} c_{3}$ and $c_{1} p>0, c_{4} p>0$ (or $c_{1} c_{4}<c_{2} c_{3}$ and $c_{1} p<0, c_{4} p<0$ ), these functions obviously represent wedge-shaped kinks in the $x t_{1}$-plane, see also Fig. 2. Choosing $p>0$ and switching on a negative $q$, these wedges become localized and, around certain negative values of $q$, then take the dromion form.

Fig. 3 shows plots of a two-dromion solution determined by (5.7) with $L=\operatorname{diag}\left(3 I_{2}, 2 I_{2}\right)$, $R=\operatorname{diag}\left(-2 I_{2},-(3 / 2) I_{2}\right)$, and $^{10}$

$$
C=\left(\begin{array}{cccc}
1 & \frac{1}{2} & 0 & 0 \\
0 & 1 & 0 & 0 \\
0 & 0 & 2 & 1 \\
0 & 0 & 5 & 3
\end{array}\right) .
$$

The diagonal $2 \times 2$ blocks of these matrices correspond to matrix data of single dromions. Such a superposition is obtained for any two solutions, provided that off-diagonal blocks of the

\footnotetext{
${ }^{10}$ If all lower-diagonal entries of $C$ are zero, we obtain $v=0$ and thus a solution of the linear equations (3.12). The plots are surprisingly insensitive with respect to changes in this range of parameters, as long as the offdiagonal entries in a diagonal block are not all close to zero and the determinant of the block is not close to zero.
} 

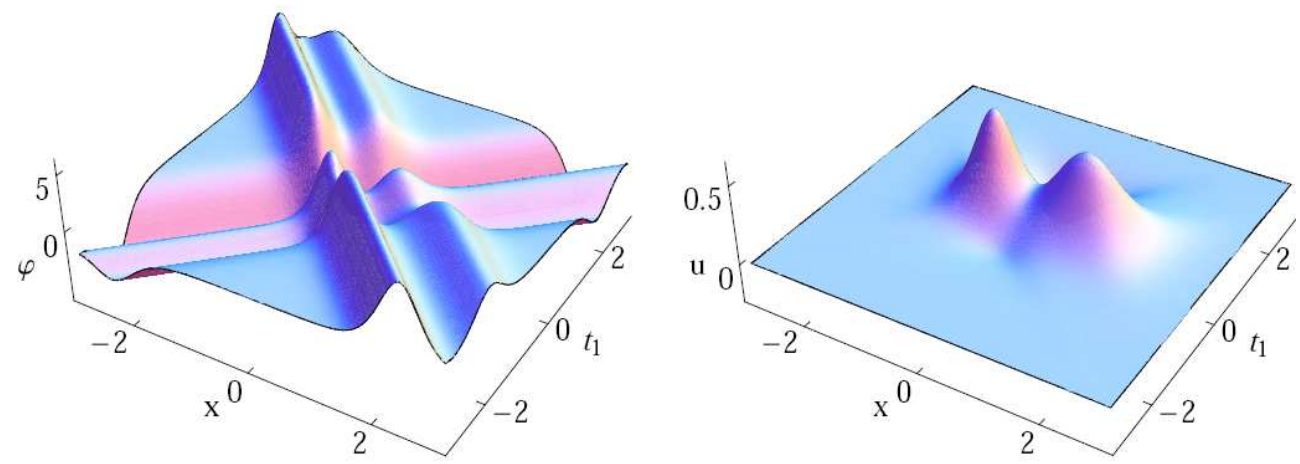

Figure 3. A two-dromion solution of (3.10) and (3.11) at $t_{2}=1$, determined by the data specified in Example 3. The left plot shows $\varphi=s_{t_{1}}=\operatorname{tr}(\phi)_{t_{1}}$.
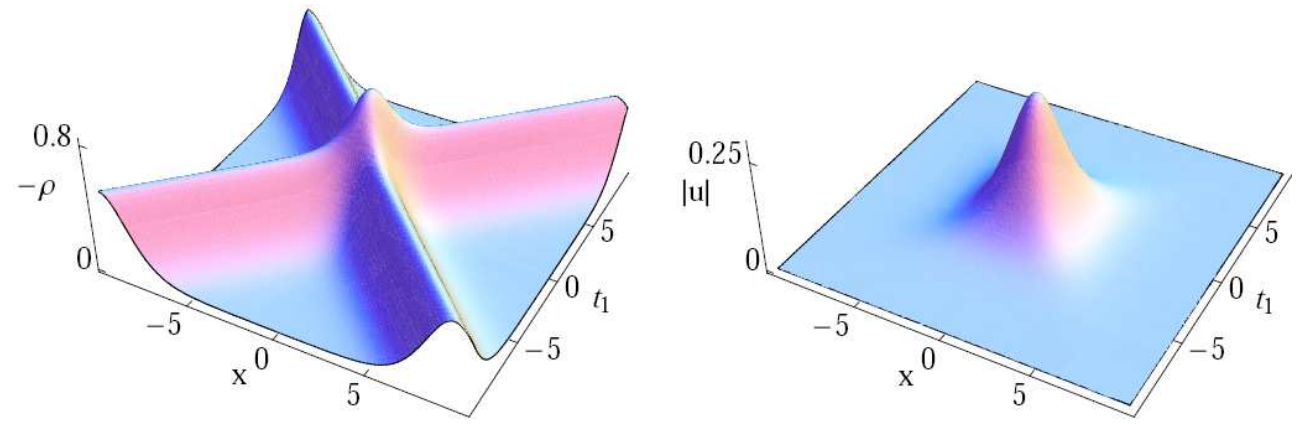

Figure 4. A dromion solution of the Davey-Stewartson-I equation with $\epsilon=-1$ at $t_{2}=0$, see the end of Example 3. Here we chose $p_{1}=p_{2}=(1+\mathrm{i}) / \sqrt{2}, q_{1}=-q_{2}=(1-\mathrm{i}) / \sqrt{2}, c_{1}=-c_{2}=\mathrm{i} \sqrt{2}, c_{3}=c_{4}=\sqrt{2}$. Here $\rho$ is given by (3.17).

matrix $K$ exist such that (5.6) can be satisfied. This is so in the restricted case considered above (see (5.7) and (5.8)), which in particular leads to multi-dromion solutions. Introducing non-zero constants in the off-diagonal blocks of $C$, leads to solutions with more complicated behaviour.

Setting $t_{n}=0, n>2$, the transition to the Davey-Stewartson system (3.18) with $\kappa=1$, which is the DS-I case, is given by the transformation of independent variables

$$
x=\frac{1+\mathrm{i}}{2 \sqrt{2}}(z-\mathrm{i} y), \quad t_{1}=-\frac{1-\mathrm{i}}{2 \sqrt{2}}(z+\mathrm{i} y), \quad t_{2}=-\mathrm{i} t .
$$

The dependent variables are $u$ and $\rho$, the latter given by (3.17). We have to take the additional constraint $v=\mathrm{i} \epsilon \bar{u}$ into account (see Example 2). One recovers a DS-I dromion within the class of solutions restricted by $q_{1}=\bar{p}_{1}, q_{2}=-\bar{p}_{2}, c_{1}$ imaginary and $c_{4}$ real, and $c_{3}= \pm \mathrm{i} \bar{c}_{2}$ with sign corresponding to $\epsilon= \pm 1$. Fig. 4 shows an example.

Case 2. Let $M=N, R=L, S=0$, and

$$
Q=J+[L, K],
$$

with constant $N \times N$ matrices $K$ and $J$ (over $\mathcal{A}$ ) that commute with all $B \in \mathcal{B}$. Furthermore, $J$ has to commute with $L$, i.e. $[J, L]=0$. Then

$$
\Phi=e^{\xi(x, \mathbf{t} ; L, B)} C e^{-\xi(x, \mathbf{t} ; L, B)}\left(I_{N}+\left(\xi^{\prime}(x, \mathbf{t} ; L, B) J-K\right) e^{\xi(x, \mathbf{t} ; L, B)} C e^{-\xi(x, \mathbf{t} ; L, B)}\right)^{-1},
$$

where $C$ is an arbitrary constant $N \times N$ matrix (with entries in $\mathcal{A}$ ) and

$$
\xi^{\prime}(x, \mathbf{t} ; L, B):=x+\sum_{B \in \mathcal{B}} \sum_{n=1}^{\infty} n t_{B, n} L^{n-1} B^{n} .
$$


If also $Q=V U^{\top}$ with vectors $U, V$ that commute with all $B \in \mathcal{B}$, then $\phi=U^{\top} \Phi V$ solves the mcKP hierarchy in $\mathcal{A}$. It remains to solve

$$
J+[L, K]=V U^{\top} .
$$

A natural choice for $J$ is the unit matrix $I_{N}$, but there are others. (5.15) can also be written as

$$
\Phi=\left(e^{\xi(x, \mathbf{t} ; L, B)} \tilde{C} e^{-\xi(x, \mathbf{t} ; L, B)}+\xi^{\prime}(x, \mathbf{t} ; L, B) J-K\right)^{-1}
$$

with an arbitrary constant $N \times N$ matrix $\tilde{C}{ }^{11}$ If $\tilde{C}$ is chosen such that it commutes with $L$ and $B$, then $\Phi$ and the corresponding solution $\phi$ of the mcKP hierarchy are purely rational functions of the independent variables. A localized solution of this kind, hence with rational decay, is usually called a "lump". The following example in particular demonstrates that there can be weaker conditions that lead to solutions with rational decay.

Example 4. Choosing $B=\operatorname{diag}(1,-1), L=\operatorname{diag}\left(p_{1}, p_{2}\right)$ and $Q=I_{2}$, (5.14) is solved by $K=\operatorname{diag}\left(k_{1}, k_{2}\right)$. Expressing $C$ again as in (5.10), we find

$$
e^{\xi(x, \mathbf{t} ; L, B)} C e^{-\xi(x, \mathbf{t} ; L, B)}=\left(\begin{array}{cc}
c_{1} & c_{2} e^{\xi_{+}\left(x, \mathbf{t} ; p_{1}\right)-\xi-\left(x, \mathbf{t} ; p_{2}\right)} \\
c_{3} e^{-\xi_{+}\left(x, \mathbf{t} ; p_{1}\right)+\xi_{-}\left(x, \mathbf{t} ; p_{2}\right)} & c_{4}
\end{array}\right),
$$

with $\xi_{ \pm}(x, \mathbf{t} ; p)$ defined in $(5.9)$, and $\xi^{\prime}(x, \mathbf{t} ; L, B)=\operatorname{diag}\left(\xi_{+}^{\prime}\left(x, \mathbf{t} ; p_{1}\right), \xi_{-}^{\prime}\left(x, \mathbf{t} ; p_{2}\right)\right)$, where

$$
\xi_{ \pm}^{\prime}(x, \mathbf{t} ; p):=x+\sum_{n=1}^{\infty} 2 n t_{2 n} p^{2 n-1} \pm \sum_{n=0}^{\infty}(2 n+1) t_{2 n+1} p^{2 n}
$$

Then (5.15) leads to the following solution of (3.3), with $B=\operatorname{diag}(1,-1)$, and its hierarchy,

$$
\phi(x, \mathbf{t})=\frac{1}{\mathcal{D}(x, \mathbf{t})}\left(\begin{array}{cc}
c_{1}+d\left(\xi_{-}^{\prime}\left(x, \mathbf{t} ; p_{2}\right)-k_{2}\right) & c_{2} e^{\xi_{+}\left(x, \mathbf{t} ; p_{1}\right)-\xi_{-}\left(x, \mathbf{t} ; p_{2}\right)} \\
c_{3} e^{-\xi+\left(x, \mathbf{t} ; p_{1}\right)+\xi-\left(x, \mathbf{t} ; p_{2}\right)} & c_{4}+d\left(\xi_{+}^{\prime}\left(x, \mathbf{t} ; p_{1}\right)-k_{1}\right)
\end{array}\right),
$$

where $d:=c_{1} c_{4}-c_{2} c_{3}$ and

$$
\begin{aligned}
\mathcal{D}(x, \mathbf{t}):= & 1+c_{1}\left(\xi_{+}^{\prime}\left(x, \mathbf{t} ; p_{1}\right)-k_{1}\right)+c_{4}\left(\xi_{-}^{\prime}\left(x, \mathbf{t} ; p_{2}\right)-k_{2}\right) \\
& +d\left(\xi_{+}^{\prime}\left(x, \mathbf{t} ; p_{1}\right)-k_{1}\right)\left(\xi_{-}^{\prime}\left(x, \mathbf{t} ; p_{2}\right)-k_{2}\right) .
\end{aligned}
$$

The components $u=\phi_{1,2}$ and $v=\phi_{2,1}$, together with

$$
s=\operatorname{tr}(\phi)=\frac{d}{\mathcal{D}}\left(\xi_{+}^{\prime}\left(x, \mathbf{t} ; p_{1}\right)+\xi_{-}^{\prime}\left(x, \mathbf{t} ; p_{2}\right)+c\right),
$$

with a constant $c$, thus solve the system (3.10), (3.11). For $c_{3}=0$, this determines a solution of the linear equations (3.12).

The transition to the Davey-Stewartson system (3.18) with $\kappa=\mathrm{i}$, which is the DS-II case, involves the transformation of independent variables

$$
x=\frac{1+\mathrm{i}}{2 \sqrt{2}}(y+z), \quad t_{1}=\frac{1-\mathrm{i}}{2 \sqrt{2}}(y-z), \quad t_{2}=-\mathrm{i} t .
$$

We set $t_{n}=0, n>2$, in the following. The lump solution of the DS-II equation [43, 44, 17] (see also $[45,46,40,47])$ is obtained as follows from the above formula. Besides taking account of the constraint $v=\mathrm{i} \epsilon \bar{u}$, we have to arrange in particular that the exponential in $u$ becomes a phase

\footnotetext{
${ }^{11}$ In the transition from (5.15) to (5.17), one assumes that $C$ is invertible with inverse $\tilde{C}$. But $\tilde{C}$ need not be invertible in order that (5.17) determines a solution of the $\operatorname{mcKP}_{Q}$ hierarchy.
} 
factor (up to some constant factor), i.e. the real part of its exponent has to be constant. This requires setting $p_{2}=-\mathrm{i} \bar{p}_{1}$. It is more tricky to find conditions on the remaining parameters such that $\mathcal{D} \neq 0$ for all $y, z, t$, so that the solution is regular. Choosing

$$
c_{4}=-\mathrm{i} \bar{c}_{1}, \quad c_{3}=\mathrm{i} \bar{c}_{2}, \quad k_{2}=-\mathrm{i} \bar{k}_{1},
$$

and renaming $k_{1}, p_{1}$ to $k, p$, we find that

$$
\mathcal{D}=\frac{1}{4}\left(\left|c_{1}\right|^{2}+2\left|c_{2}\right|^{2}\right)\left(\left(y-y_{0}-v_{y} t\right)^{2}+\left(z-z_{0}-v_{z} t\right)^{2}\right)+\frac{2\left|c_{2}\right|^{2}}{\left|c_{1}\right|^{2}+2\left|c_{2}\right|^{2}},
$$

where $v_{y}=-2 \operatorname{Im}(p), v_{z}=2 \operatorname{Re}(p)$, and

$$
y_{0}=\operatorname{Re}(k)-\frac{2 \operatorname{Re}\left(c_{1}\right)}{\left|c_{1}\right|^{2}+2\left|c_{2}\right|^{2}}, \quad z_{0}=\operatorname{Im}(k)+\frac{2 \operatorname{Im}\left(c_{1}\right)}{\left|c_{1}\right|^{2}+2\left|c_{2}\right|^{2}} .
$$

The resulting DS-II solution

$$
\begin{aligned}
& u=\frac{c_{2}}{\mathcal{D}} e^{\mathrm{i}\left(\operatorname{Im}(p) y+\operatorname{Re}(p) z-\operatorname{Re}\left(p^{2}\right) t\right),} \\
& \rho=\frac{1}{4 \mathcal{D}^{2}}\left(\left(\left|c_{1}\right|^{2}+2\left|c_{2}\right|^{2}\right)^{2}\left(\left(z-z_{0}-v_{z} t\right)^{2}-\left(y-y_{0}-v_{y} t\right)^{2}\right)-8\left|c_{2}\right|^{2}\right),
\end{aligned}
$$

with $\rho$ defined in (3.17) and $\epsilon=1$, is regular whenever $c_{2} \neq 0$ and reproduces a well-known lump solution.

Again, (lump) solutions can be superposed by taking matrix data of (lump) solutions as diagonal blocks of larger matrices $L$ and $C$. It then essentially remains to determine the offdiagonal blocks of the new matrix $K$ so that (5.16) holds.

\section{The matrix Riccati system associated with the multicomponent KP hierarchy}

Writing

$$
H^{n}=:\left(\begin{array}{cc}
R_{n} & Q_{n} \\
S_{n} & L_{n}
\end{array}\right)
$$

the matrix linear system (5.1), (5.2) implies the matrix Riccati system

$$
\begin{aligned}
& \Phi_{x}=S+L \Phi-\Phi R-\Phi Q \Phi, \\
& \Phi_{t_{B, n}}=B^{n} S_{n}+B^{n} L_{n} \Phi-\Phi B^{n} R_{n}-\Phi B^{n} Q_{n} \Phi .
\end{aligned}
$$

The two solution families presented in Section 5 solve this matrix Riccati system, with the respective conditions imposed on the matrices $L, Q, R, S$.

Abstracting from matrices and thinking of $L, R, S$ as (noncommutative) algebraic objects, their elimination from the above system leads to the mcKP hierarchy with product modified by $Q$ (cf. [48]). To some extent the above Riccati system thus expresses the mcKP hierarchy as a hierarchy of ordinary differential equations.

Finite-size matrix Riccati equations, in particular with constant coefficient matrices as above, were discussed in a context related to integrable systems already long ago (see e.g. [49, 50]), but apparently not in the context of the KP hierarchy. A special infinite-size matrix Riccati system involving the shift operator in infinite dimensions appeared, however, in the framework of the Sato theory (see e.g. $[51,13]$ ). In the one-component case with $B=I$, the above Riccati system, with suitable conditions imposed on the coefficient matrices, also generates solutions of the BKP and the CKP hierarchy [52]. The Riccati system indeed generates solutions of various integrable systems and therefore deserves to be studied in its own right. 
Remark 3. For fixed $r \in \mathbb{N}, r>1$, and for some fixed $B \in \mathcal{B}$, let us consider the condition

$$
(H B)^{r} Z_{0}=Z_{0} P
$$

with an $N \times N$ matrix $P$ (over $\mathcal{A}$ ). For the solution (5.3) of the linear matrix system (5.2), this implies $(H B)^{n r} Z=Z P^{n}$ for $n \in \mathbb{N}$, hence $B^{n r}\left(R_{n r} X+Q_{n r} Y\right)=X P^{n}$ and $B^{n r}\left(S_{n r} X+L_{n r} Y\right)=$ $Y P^{n}$, and thus the algebraic Riccati equations $B^{n r}\left(S_{n r}+L_{n r} \Phi\right)=Y P^{n} X^{-1}=\Phi X P^{n} X^{-1}=$ $\Phi B^{n r}\left(R_{n r}+Q_{n r} \Phi\right)$. The corresponding equations (6.2) of the Riccati system then read

$$
\Phi_{t_{B, n r}}=B^{n r}\left(S_{n r}+L_{n r} \Phi\right)-\Phi B^{n r}\left(R_{n r}-Q_{n r} \Phi\right)=0, \quad n=1,2, \ldots
$$

Hence $\phi$ solves the $(r, B)$-reduction, i.e. the $r$-reduction (multicomponent version of $r$ th GelfandDickey hierarchy) with respect to $B$.

\section{Conclusions}

Any solution of a multicomponent Burgers (mcBurgers) hierarchy is a solution of the corresponding multicomponent KP (mcKP) hierarchy. Furthermore, there is a functional equation that determines the mcKP hierarchy and has the form of an inhomogeneous mcBurgers hierarchy functional equation. We have also shown that the mcKP linear system is equivalent to a mcBurgers hierarchy, where the dependent variable has the structure of an infinite Frobenius companion matrix (which in particular makes contact with [9]).

Moreover, we have shown how solutions of a mcKP hierarchy are obtained from solutions of a multicomponent linear heat hierarchy via a generalized Cole-Hopf transformation. An important subcase generates solutions of a mcKP hierarchy from solutions of a matrix linear system and we presented some explicit solution formulae. They comprise in particular DaveyStewartson dromions and lump solutions.

There is certainly a lot more to be (re)discovered using the rather simple but quite general method in Section 5 to construct exact solutions, but we are far from a systematic way to explore the properties of solutions obtained in this way. Furthermore, we have stressed the role of a matrix Riccati hierarchy in this context.

\section{References}

[1] Hopf E., The partial differential equation $u_{t}+u u_{x}=\mu u_{x x}$, Comm. Pure Appl. Math. 3 (1950), 201-230.

[2] Cole J.D., On a quasi-linear parabolic equation occurring in aerodynamics, Quart. Appl. Math. 9 (1951), $225-236$.

[3] Sawada K., Kotera T., A method for finding $N$-soliton solutions of the K.d.V. equation and K.d.V.-like equation, Progr. Theoret. Phys. 51 (1974), 1355-1367.

[4] Mikhailov A., Shabat A., Sokolov V., The symmetry approach to classification of integrable equations, in What Is Integrability?, Editor V. Zakharov, Springer Ser. Nonlinear Dynam., Springer, Berlin, 1991, $115-184$.

[5] Guil F., Mañas M., Álvarez G., The Hopf-Cole transformation and the KP equation, Phys. Lett. A 190 (1994), 49-52.

[6] Guil F., Mañas M., The Dirac equation and integrable systems of KP type, J. Phys. A: Math. Gen. 29 (1996), 641-665.

[7] Dimakis A., Müller-Hoissen F., Burgers and KP hierarchies: a functional representation approach, Theoret. and Math. Phys. 152 (2007), 933-947, nlin.SI/0610045.

[8] Dimakis A., Müller-Hoissen F., With a Cole-Hopf transformation to solutions of the noncommutative KP hierarchy in terms of Wronski matrices, J. Phys. A: Math. Theor. 40 (2007), F321-F329, nlin.SI/0701052. 
[9] Zenchuk A.I., Santini P.M., The remarkable relations among PDEs integrable by the inverse spectral transform method, by the method of characteristics and by the Hopf-Cole transformation, J. Phys. A: Math. Theor. 41 (2008), 185109, 28 pages, arXiv:0801.3945.

[10] Dimakis A., Müller-Hoissen F., Nonassociativity and integrable hierarchies, nlin.SI/0601001.

[11] Dimakis A., Müller-Hoissen F., Weakly nonassociative algebras, Riccati and KP hierarchies, in Generalized Lie Theory in Mathematics, Physics and Beyond, Editors S. Silvestrov, E. Paal, V. Abramov and A. Stolin, Springer, Berlin, 2008, 9-27, nlin.SI/0701010.

[12] Sato M., Sato Y., Soliton equations as dynamical systems on infinite dimensional Grassmann manifold, in Nonlinear Partial Differential Equations in Applied Science, Editors H. Fujita, P.D. Lax and G. Strang, North-Holland Math. Stud., Vol. 81, North-Holland, Amsterdam, 1982, 259-271.

[13] Takasaki K., Geometry of universal Grassmann manifold from algebraic point of view, Rev. Math. Phys. 1 (1989), 1-46.

[14] Davey A., Stewartson K., On three-dimensional packets of surface waves, Proc. Roy. Soc. London Ser. A 338 (1974), 101-110.

[15] Anker D., Freeman N.C., On the soliton solutions of the Davey-Stewartson equation for long waves, Proc. Roy. Soc. London Ser. A 360 (1978), 529-540.

[16] Benney D., Roskes G., Wave instabilities, Stud. Appl. Math. 48 (1969), 377-385.

[17] Nakamura A., General superposition of solitons and various ripplons of a two-dimensional nonlinear Schrödinger equation, J. Math. Phys. 23 (1982), 1422-1426.

[18] Sato M., Soliton equations as dynamical systems on infinite dimensional Grassmann manifolds, in Random Systems and Dynamical Systems (Kyoto, 1981), RIMS Kokyuroku 439 (1981), 30-46.

[19] Date E., Jimbo M., Kashiwara M., Miwa T., Transformation groups for soliton equations. III. Operator approach to the Kadomtsev-Petviashvili equation, J. Phys. Soc. Japan 50 (1981), 3806-3812.

[20] Kajiwara K., Matsukidaira J., Satsuma J., Conserved quantities of two-component KP hierachy, Phys. Lett. B 146 (1990), 115-118.

[21] Oevel W., Darboux theorems and Wronskian formulas for integrable systems. I. Constrained KP flows, Phys. A 195 (1993), 533-576.

[22] Bergvelt M.J., ten Kroode A.P.E., Partitions, vertex operator constructions and multi-component KP equations, Pacific J. Math. 171 (1995), 23-88, hep-th/9212087.

[23] Doliwa A., Mañas M., Martinez Alonso L., Medina E., Santini P.M., Charged free fermions, vertex operators and the classical theory of conjugate nets, J. Phys. A: Math. Gen. 32 (1999), 1197-1216, solv-int/9803015.

[24] Dickey L., Soliton equations and Hamiltonian systems, 2nd ed., Advanced Series in Mathematical Physics, Vol. 26, World Scientific Publishing Co., Inc., River Edge, NJ, 2003.

[25] Kac V.G., van der Leur J.W., The n-component KP hierarchy and representation theory, J. Math. Phys. 44 (2003), 3245-3293, hep-th/9308137.

[26] Kundu A., Strampp W., Derivative and higher-order extensions of Davey-Stewartson equation from matrix Kadomtsev-Petviashvili hierarchy, J. Math. Phys. 36 (1995), 4192-4202, hep-th/9311153.

[27] Bogdanov L.V., Konopelchenko B.G., Analytic-bilinear approach to integrable hierarchies. II. Multicomponent KP and 2D Toda lattice hierarchies, J. Math. Phys. 39 (1998), 4701-4728, solv-int/9609009.

[28] Cheng Y., Li Y.S., Constraints of the $2+1$ dimensional integrable soliton systems, J. Phys. A: Math. Gen. 25 (1992), 419-431.

[29] Konopelchenko B., Strampp W., New reductions of the Kadomtsev-Petviashvili and two-dimensional Toda lattice hierarchies via symmetry constraints, J. Math. Phys. 33 (1992), 3676-3686.

[30] Yurov A., Bäcklund-Schlesinger transformations for Davey-Stewartson equations, Theoret. and Math. Phys. 109 (1996), 1508-1514.

[31] Leznov A.N., Yuzbashyan E.A., Multi-soliton solutions of the two-dimensional matrix Davey-Stewartson equation, Nuclear Phys. B 496 (1997), 643-653, hep-th/9612107.

[32] Boiti M., Leon J.-P., Martina L., Pempinelli F., Scattering of localized solitons in the plane, Phys. Lett. A 132 (1988), 432-439.

[33] Fokas A.S., Santini P.M., Coherent structures in multidimensions, Phys. Rev. Lett. 63 (1989), $1329-1333$.

[34] Santini P.M., Energy exchange of interacting coherent structures in multidimensions, Phys. D 41 (1990), $26-54$. 
[35] Hietarinta J., Hirota R., Multidromion solutions to the Davey-Stewartson equation, Phys. Lett. A 145 (1990), 237-244.

[36] Jaulent M., Manna M., Martinez-Alonso L., Fermionic analysis of Davey-Stewartson dromions, Phys. Lett. A 151 (1990), 303-307.

[37] Nakao T., Wadati M., Davey-Stewartson equation and properties of dromion, J. Phys. Soc. Japan 62 (1993), 933-947.

[38] Sall' M.A., The Davey-Stewartson equations, J. Math. Sci. 68 (1994), 265-270.

[39] Gilson C.R., Nimmo J.J.C., A direct method for dromion solutions of the Davey-Stewartson equations and their asymptotic properties, Proc. Roy. Soc. London Ser. A 435 (1991), 339-357.

[40] Pelinovsky D., On a structure of the explicit solutions to the Davey-Stewartson equations, Phys. D 87 (1995), 115-122.

[41] Guil F., Mañas M., Deformation of the dromion and solitoff solutions of the Davey-Stewartson I equation, Phys. Lett. A 209 (1995), 39-47.

[42] Hietarinta J., One-dromion solutions for generic classes of equations, Phys. Lett. A 149 (1990), $113-118$.

[43] Satsuma J., Ablowitz M.J., Two-dimensional lumps in nonlinear dispersive systems, J. Math. Phys. 20 (1979), 1496-1503.

[44] Nakamura A., Explode-decay mode lump solitons of a two-dimensional nonlinear Schrödinger equation, Phys. Lett. A 88 (1982), 55-56.

[45] Fokas A.S., Ablowitz M.J., Method of solution for a class of multidimensional nonlinear evolution equations, Phys. Rev. Lett. 51 (1983), 7-10.

[46] Arkadiev V., Pogrebkov A., Polivanov M., Inverse scattering transform method and soliton solutions for Davey-Stewartson II equation, Phys. D 36 (1989), 189-197.

[47] Mañas M., Santini P.M., Solutions of the Davey-Stewartson II equation with arbitrary rational localization and nontrivial interaction, Phys. Lett. A 227 (1997), 325-334.

[48] Dimakis A., Müller-Hoissen F., A new approach to deformation equations of noncommutative KP hierarchies, J. Phys. A: Math. Theor. 40 (2007), 7573-7596, math-ph/0703067.

[49] Winternitz P., Lie groups and solutions of nonlinear partial differential equations, in Nonlinear Phenomena (Oaxtepec, 1982), Lecture Notes in Phys., Vol. 189, Springer, Berlin, 1983, 263-331.

[50] del Olmo M.A., Rodriguez M., Winternitz P., Superposition formulas for rectangular matrix Riccati equations, J. Math. Phys. 28 (1987), 530-535.

[51] Dorfmeister J., Neher E., Szmigielski J., Automorphisms of Banach manifolds associated with the KPequation, Quart. J. Math. Oxford Ser. (2) 40 (1989), 161-195.

[52] Dimakis A., Müller-Hoissen F., BKP and CKP revisited: The odd KP system, arXiv:0810.0757. 